Check for updates

Cite this: RSC Adv., 2017, 7, 25872

Accepted 7th May 2017

DOI: $10.1039 / c 7 r a 02010 d$

rsc.li/rsc-advances
Received 17th February 2017

\section{Molecular analysis of polysaccharide accumulation in Dendrobium nobile infected with the mycorrhizal fungus Mycena sp. $\dagger$}

\begin{abstract}
Qing Li, (D) Bing Li, Li-Si Zhou, Gang Ding, Biao Li* and Shun-Xing Guo*
Polysaccharides are among the most important active compounds of Dendrobium nobile Lindl., and they improve immunity, strengthen memory, and prevent and fight tumours. Evidence suggests that the mycorrhizal fungus Mycena sp. may increase the polysaccharide content of $D$. nobile. To understand the regulatory mechanism by which Mycena sp. promotes polysaccharide accumulation in $D$. nobile, a model including MF23 (Mycena sp.) and D. nobile was developed. Content measurements and electron microscopy showed that the change in polysaccharides was associated with the level of colonization. Large-scale transcriptome sequencing analyses of D. nobile infected with MF23 and of wild D. nobile revealed that 30 unigenes encoding key enzymes were potentially associated with polysaccharide accumulation. A qRT-PCR experiment involving 13 randomly chosen unigenes suggested that the combined effects of the carbohydrate biosynthesis and consumption pathways resulted in changes in polysaccharide levels. MF23 might increase polysaccharide level by inducing genes involved in photosynthesis (petF), starch and sucrose metabolism (SUS, bcSA, glgA) and fructose and mannose metabolism (PMM, GPMM) and by repressing genes involved in glycolysis ( $P G K, P F K)$ and the citrate cycle $(P D H B, C S, S D H A)$. This study provides a good example of an endophyte promoting the synthesis of bioactive compounds in its host and paves the way for further investigations of the associated regulatory mechanism using molecular methods.
\end{abstract}

\section{Introduction}

Dendrobium nobile Lindl. is an endangered orchid species that is distributed in India, Burma, Thailand, Laos, Vietnam, and China. ${ }^{1}$ For thousands of years, its stem has been used as a tonic herb in traditional medicine, and its remarkable curative effects on gastritis, diabetes, aging and immunological disorders have been proven by modern pharmacological research. ${ }^{2-6}$ Thus, it has been officially listed as "Shi Hu" in different versions of the Pharmacopoeia of the People's Republic of China. To elucidate the pharmacological mechanism of D. nobile, substantial research has been performed on its compounds, and these studies have demonstrated that polysaccharides are among the most important active compounds of D. nobile..$^{7-9}$ In addition to their effects on strengthening memory ${ }^{10}$ and inhibiting thrombokinesis, ${ }^{\mathbf{1 1}}$ many polysaccharides derived from $D$. nobile exhibit strong immunomodulatory and antitumour activities. ${ }^{\mathbf{1 2 - 1 4}}$ For instance, the polysaccharide DNP4-2 can significantly increase the immune index, strongly promote the

Institute of Medicinal Plant Development, Peking Union Medical College, Chinese Academy of Medical Sciences, Beijing 100193, People's Republic of China. E-mail: libiao@126.com; sxguo1986@163.com

† Electronic supplementary information (ESI) available. See DOI: 10.1039/c7ra02010d secretion of tumour necrosis factor alpha (TNF- $\alpha$ ), interferongamma (IFN- $\gamma$ ) and interleukin-2 (IL-2), and reduce the concentrations of malondialdehyde (MDA) in the serum. ${ }^{15}$ At $200 \mu \mathrm{g} \mathrm{mL}{ }^{-1}$, the polysaccharides DNP-W1 and DNP-W3 show strong antitumour activity (antitumour rate $>80 \%$ ), ${ }^{16}$ suggesting that the polysaccharides of $D$. nobile could be effective natural antitumour agents. However, the content of polydevelopment and utilization.

Orchids are typical mycorrhizal plants. Several photosynthetic orchids have recently been confirmed as mixotrophic, with the ability to recover carbon from mycorrhizal fungi associated with their roots. ${ }^{18}$ Mycorrhizal orchids can acquire more N, P and water than non-mycorrhizal controls. ${ }^{19,20}$ To date, our laboratory has isolated numerous mycorrhizal fungi with growth-promoting effects from the roots of wild $D$. officinale Kimura et Migo and D. nobile. ${ }^{\mathbf{2 1 , 2 2}}$ In addition, some mycorrhizal fungi have been reported to promote polysaccharide accumulation in their hosts. In D. nobile infected with Mycena sp., the polysaccharide level increased over a longer period ${ }^{23}$ and polysaccharides accumulated to much higher levels than those of controls (76.7\% increase). ${ }^{24}$ However, the relationship between mycorrhizal fungi and their hosts is still not clear, which hinders the application of mycorrhizal fungi. Thus, it is of great importance to understand the influence and regulatory saccharides in D. nobile is not high, ${ }^{17}$ which restricts their 
mechanism of mycorrhizal fungi on the polysaccharide metabolism of $D$. nobile.

The accumulation of polysaccharides can be affected by many factors, such as photosynthesis, ${ }^{25}$ starch and sucrose metabolism, ${ }^{26,27}$ and respiration. ${ }^{28}$ The Illumina HiSeq4000 platform was initially used to focus on the key genes in these processes and to reveal the molecular events underlying the changes in the polysaccharides of symbiotic $D$. nobile. This study provides an overview of the $D$. nobile stem transcriptome before and after inoculation. In addition, the results in this report provide a good example of an endophyte promoting the synthesis of bioactive compounds in its host and pave the way for further investigations of the associated regulatory mechanism using molecular methods.

\section{Experimental}

\section{Biological materials and culture conditions}

D. nobile explants of $3-5 \mathrm{~cm}$ in height were cultured on improved 1/2 Murashige and Skoog (MS) medium supplemented with potato juice $\left(200 \mathrm{~g} \mathrm{~L}^{-1}\right.$ potato, juice boiled for $20 \mathrm{~min}, 3 \%(\mathrm{w} / \mathrm{v})$ sucrose, and $0.6 \%(\mathrm{w} / \mathrm{v})$ agar, $\mathrm{pH}$ 5.8). Each bottle contained 9 seedlings. Mycelial plugs with a diameter of 9 $\mathrm{mm}$, which were from 20 day-old MF23 (Mycena sp.) ${ }^{29}$ grown on potato dextrose agar medium, were placed in the centre of a culture bottle, adjacent to the roots of $D$. nobile. Then, the culture bottles were kept in a conventional greenhouse with a $10 \mathrm{~h}$ light $/ 14 \mathrm{~h}$ dark photoperiod at $(24 \pm 1)^{\circ} \mathrm{C}$ and an illumination intensity of $1500 \mathrm{Lx}$. Seedlings that were not inoculated with mycelial plugs were maintained as controls.

Plant samples were collected at different stages, including 1 , 3, 6 and 9 weeks post-inoculation (model group) and at the same stages for the non-inoculated (control) group. All samples were divided into two portions; some were used for morphological and chemical research, and the rest were frozen using liquid nitrogen and stored until RNA extraction.

\section{Determination of the polysaccharide content of $D$. nobile}

Stems of each treatment were dried at $55{ }^{\circ} \mathrm{C}$, thoroughly blended, and ground to a powder. The polysaccharide extraction method was as in Chen et al. ${ }^{30}$ The polysaccharide content was determined using the phenol-sulfuric acid method described by the Pharmacopoeia Committee of the People's Republic of China ${ }^{31}$ and Dubois et al. ${ }^{32}$ with glucose solutions $\left(0,5,10,20,30,40,50,60,70\right.$, and $\left.80 \mu \mathrm{g} \mathrm{mL}^{-1}\right)$ as standards. The linear regression equation was $y=7.6021 x+0.0609(r=$ 0.9998).

\section{Light microscopy}

Fresh root segments were fixed in formalin-acetic acid-alcohol (FAA), an approach described by Feder and O'Brien. ${ }^{33}$ Samples were dehydrated in a graded ethanol series, embedded in paraffin, stained with safranine and fast green, sealed with Gel Damar and then observed and photographed on a light microscope equipped with a camera (ZEISS Axio Imager A1).

\section{RNA isolation and sequencing}

Total RNA was extracted from 1 week-old and 9 week-old stem samples of $D$. nobile using an RNeasy Plant Mini Kit (Qiagen, Hilden, Germany) following the manufacturer's instructions. The quality and concentration of RNA were determined using electrophoresis and a Bioanalyzer 2100 (Agilent Technologies, Santa Clara, CA, USA). The best RNA samples, which had an RIN above 8.0, were chosen for cDNA library preparation.

Library construction and sequencing were performed by the Novogene Bioinformatics Institute (Beijing, China), and the general experimental pipeline is summarized in previously published papers. $^{34,35}$ Sequencing libraries were generated using an NEBNext ${ }^{\circledR}$ Ultra $^{\mathrm{TM}}$ RNA Library Prep Kit for Illumina ${ }^{\circledR}$ (NEB, USA) following the manufacturer's recommendations, and index codes were added to attribute the sequences to each sample. The clustering of the index-coded samples was performed on a cBot Cluster Generation System using a TruSeq PE Cluster Kit v3-cBot-HS (Illumina). After cluster generation, the library preparations were sequenced on an Illumina HiSeq 4000 instrument with 150 bp paired-end reads.

\section{Transcriptome assembly and annotation}

Clean reads were obtained from the raw data by removing adapter-containing reads, poly- $N$-containing reads and lowquality reads. All downstream analyses were based on highquality clean data.

Transcriptome assembly was processed using Trinity ${ }^{36}$ with min_kmer_cov set to 2 . The longest transcript from each gene was designated a unigene. To assign predicted gene descriptions for the assembled unigenes, a BLASTx search was performed against the following databases: $\mathrm{Nr}$ (NCBI nonredundant protein sequences), Nt (NCBI non-redundant nucleotide sequences), Pfam (protein family), KOG (eukaryotic orthologous groups of proteins), Swiss-Prot (a manually annotated and reviewed protein sequence database), KO (KEGG orthologue database) and GO (gene ontology), with a cutoff $E$ value of $10^{-5}$.

\section{Analysis of differentially expressed genes (DEGs)}

Differential expression analysis of model and control groups was performed using the DESeq R package (1.10.1). ${ }^{37}$ The $P$ value was adjusted using the $q$-value. ${ }^{38}$ In this study, genes with transcripts with a significant $q$-value $(<0.005)$ and $\mid \log _{2}$ (fold change) $\mid>1$ between the model and control groups were considered DEGs.

GO and KEGG enrichment analyses of the DEGs were performed using the GOseq $\mathrm{R}$ packages based on the Wallenius non-central hyper-geometric distribution ${ }^{39}$ and KOBAS $^{40}$ software, respectively.

\section{Validation and expression pattern analysis}

To experimentally validate the transcriptional abundance results from sequencing and computational analysis, 13 unigenes were selected for qRT-PCR. Primers were designed using Primer 6.0 and are listed in ESI Table 1. $\dagger$ The actin gene of $D$. 
nobile was used as a ref. 41. cDNA templates were obtained by reverse-transcribing the total RNA of the model and control groups after different lengths of time in culture. qRT-PCR was performed using SYBR® Premix Ex Taq ${ }^{\mathrm{TM}}$ (TaKaRa, Dalian, China) on a LightCycler480 II System (Roche, USA). The reaction was performed at $95{ }^{\circ} \mathrm{C}$ for $30 \mathrm{~s}$, followed by 40 cycles of $95{ }^{\circ} \mathrm{C}$ for $5 \mathrm{~s}$ and $60{ }^{\circ} \mathrm{C}$ for $30 \mathrm{~s}$. Three independent biological replicates were analysed for each sample, and three technical replicates were analysed for each biological replicate. Gene expression was evaluated using the $2^{-\Delta \Delta C t}$ method. ${ }^{42}$

\section{Statistical analysis}

SPSS 19.0 (IBM, Chicago, IL, USA) statistical software was used for the statistical evaluation of the results. All results are expressed as the mean \pm standard deviation (SD) of the number of experiments. Unpaired $t$-tests were performed at $P<0.05$.

\section{Results}

\section{Changes in the polysaccharide content of the D. nobile stem}

To understand the effect of the mycorrhizal fungus MF23 on the polysaccharide accumulation of $D$. nobile, the polysaccharide content of $D$. nobile stems at 1, 3, 6, and 9 weeks after inoculation and in the controls were assessed (Fig. 1). At the first three stages, the stems of both the model and control groups had low polysaccharide contents (less than $2.10 \%$ ). However, 9 weeks after inoculation, the concentration of polysaccharides increased to $7.11 \%$, which was 4 times greater than that of the controls.

\section{Associations between MF23 and D. nobile roots}

Light microscopy showed no hyphae in 1 week-old and control root tissues (Fig. 2A and B). The hyphae of MF23 first appeared in the velamen cells of the host roots 3 weeks after inoculation and gathered near the passage cells (Fig. 2C). With increasing time, the hyphae penetrated the cell wall of the D. nobile root cortex through the passage cells and spread from cell to cell

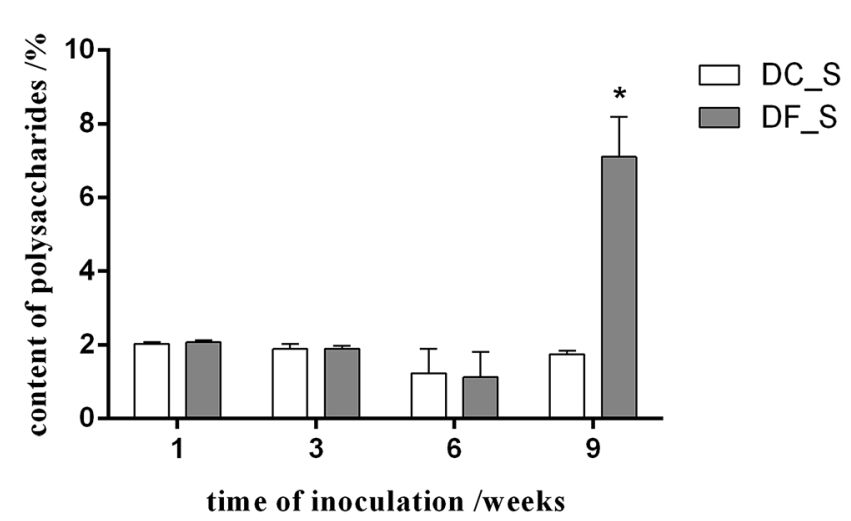

Fig. 1 The effect of MF23 on the polysaccharide content in D. nobile seedlings in tissue culture. Values are presented as the mean \pm SD $(n=$ $3)$; values with * are statistically significant at $P<0.05$. The white bar represents the control group (DC_S), and the grey bar represents the model group (DF_S).
(Fig. 2D). In the $9^{\text {th }}$ week, when the polysaccharide content of $D$. nobile had increased significantly, increasing numbers of hyphae appeared at the cortex cells of the host. In addition, pelotons, typical orchid mycorrhizal structures, ${ }^{43}$ were first observed in the passage cells (Fig. 2E). These findings suggest a possible relationship between polysaccharide accumulation and the formation of pelotons.

\section{Transcriptome profiles of inoculated and non-inoculated $D$. nobile}

To determine the $D$. nobile transcriptome expression profile after MF23 infection, next-generation sequencing was conducted on symbiotic and asymbiotic D. nobile stems 1 and 9 weeks after inoculation. In this study, two biological replicates were sequenced per sample. In total, 44863 528-69 564340 raw reads of $150 \mathrm{bp}$ were generated (Table 1 ). The raw reads are available in the NCBI SRA database under accession number PRJNA338366. After removing adaptor sequences, ambiguous nucleotides and low-quality sequences, 43312 840-67 080588 clean reads remained. The assembly of the clean reads resulted in 207283 unigenes in the range of 201-17 $019 \mathrm{bp}$, with an N50 length of $857 \mathrm{bp}$; the length distribution of the unigenes is illustrated in Fig. 3.

\section{Unigene functional annotation}

A total of 83778 (40.41\%) unigenes with significant BLAST hits were returned. Among them, 56378 (27.19\%), 34493 (16.64\%), 18911 (9.12\%), 48431 (23.36\%), 46843 (22.59\%), 47372 (22.85\%), and 27087 (13.06\%) unigenes had hits in the Nr, Nt, KO, Swiss-Prot, Pfam, GO, and KOG databases, respectively. The $E$-values and similarity distributions of the top hits in the $\mathrm{Nr}$ database analysis revealed that $36.4 \%$ and $24.9 \%$ of the genes showed significant homology $\left(E\right.$-value $\left.<10^{-45}\right)$ or high similarity (greater than $80 \%$ ), respectively (Fig. $4 \mathrm{~A}$ and B). Based on $\mathrm{Nr}$ annotation, approximately $35.2 \%$ of the annotated unigenes were assigned with the best score to sequences from the top five species, Phoenix dactylifera (13.6\%), Elaeis guineensis (13.4\%), Vitis vinifera (5.7\%), Musa acuminate (5.2\%), and Nelumbo nucifera (2.3\%; Fig. 4C).

\section{Analysis of DEGs}

To better survey the biological mechanism of polysaccharide biosynthesis induced by mycorrhizal fungi, it is important to identify the DEGs between the two different groups. Differential expression analysis was first performed between the two treatments. DEGs ( $q$-value $<0.005$ and $\mid \log _{2}$ (fold change) $\mid>1$ ) were defined as genes that were significantly enriched or depleted in one treatment relative to the other. In this study, there were a total of 1388 DEGs between DF1_S and DC1_S (1382 upregulated, 6 down-regulated). Between DF9_S and DC9_S, 2646 genes (1348 up-regulated, 1298 down-regulated) were expressed at significantly different levels. A Venn diagram shows that there were only 28 DEGs in common between DF9_S vs. DC9_S and DF1_S vs. DC1_S, implying that the expression of genes varied significantly with different stages of inoculation (Fig. 5). 


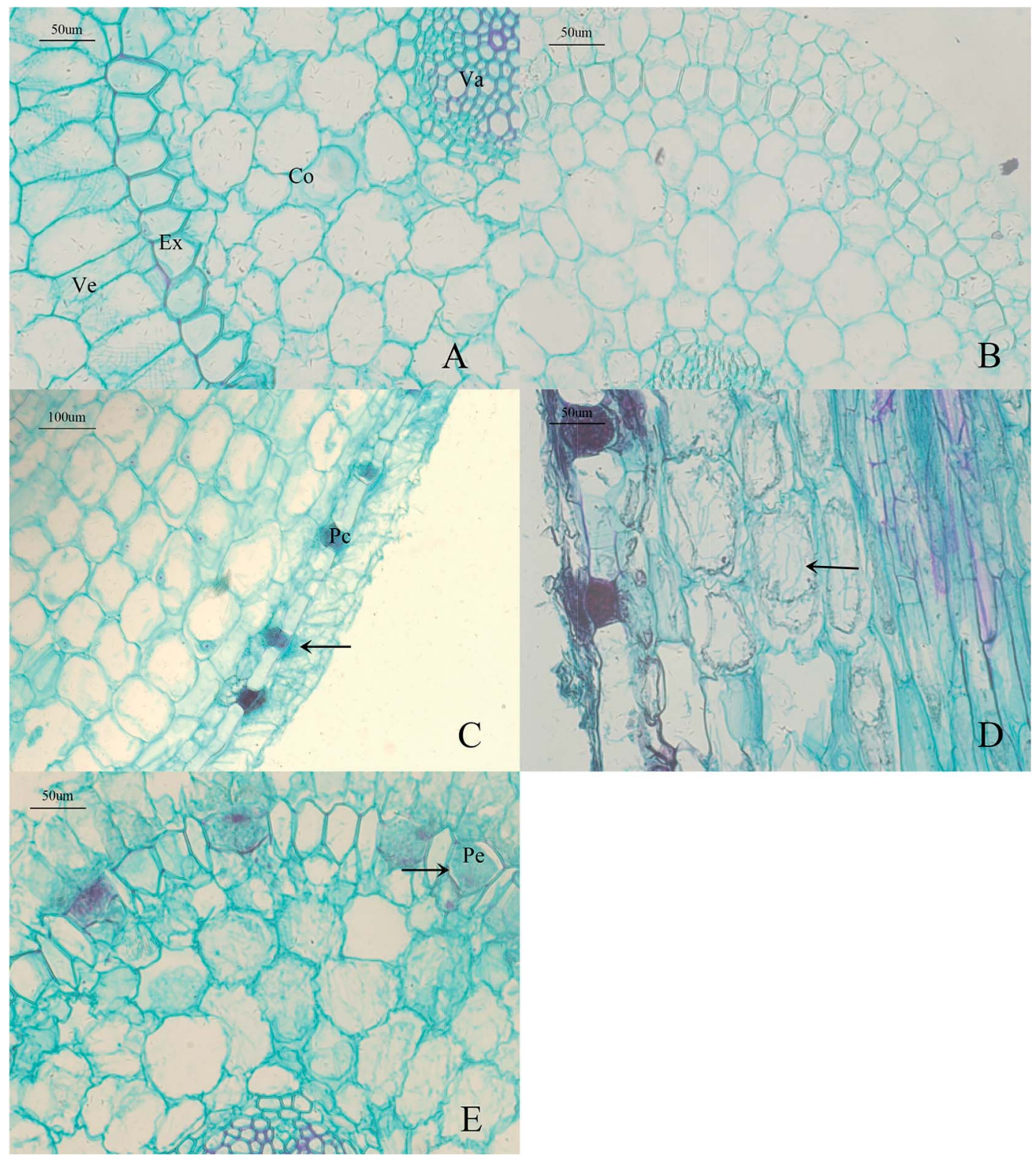

Fig. 2 Light micrographs of interactions between D. nobile roots and MF23 (A-E). (A) chemTransverse section of a control root ( $\times 20$ ). (B) Transverse section of a root after 1 week of symbiotic cultivation $(\times 10)$. (C) Vertical section of a root after 3 weeks of symbiotic cultivation $(\times 10)$. (D) Vertical section of a root after 6 weeks of symbiotic cultivation $(\times 20)$. (E) Transverse section of a root after 9 weeks of symbiotic cultivation $(\times 20)$. Co, cortex cells; Ex, exodermis; Pc, passage cells; Pe, pelotons; Va, vascular cylinder; Ve, velamen cells.

\section{Functional distribution of DEGs}

To analyse the functions of the DEGs, a GO analysis was performed. The results showed that there were 175 and 5 significantly enriched GO terms related to various biological processes for DF1_S vs. DC1_S and for DF9_S vs. DC9_S, respectively (Fig. 6). For DF1_S vs. DC1_S, 'metabolic process' (GO:0008152) was the dominant term, followed by 'cell' (GO:0005623), 'cell part' (GO:0044464), 'intracellular' (GO:0005622), 'biosynthetic process' (GO:0009058) and 'organic substance biosynthetic process' (GO:0044710). In other words, more DEGs were annotated to enriched GO terms involved in biological processes and cellular components. However, for DF9_S vs. DC9_S, the majority of DEGs were associated with DNA metabolism and substance binding, and there were no significantly enriched GO terms related to cellular components; this result was very different from that of DF1_S vs. DC1_S, implying that MF23 influenced different $D$. nobile biological processes at different stages.

$\mathrm{KEGG}^{44,45}$ is a database resource for understanding highlevel functions and utilities of the biological system from molecular-level information. KEGG pathway analyses of the DEGs from DF1_S vs. DC1_S and from DF9_S vs. DC9_S highlighted 113 and 125 pathways, respectively. The top 20 pathways of these two groups are shown in Fig. 7. Notably, among the top 20 KEGG pathways from DF1_S vs. DC1_S, 25\% were associated with polysaccharide metabolism even though 
Table 1 Sequencing output statistics

\begin{tabular}{|c|c|c|c|c|c|c|c|}
\hline Sample & Raw reads & Clean reads & $\begin{array}{l}\text { Clean } \\
\text { bases }\end{array}$ & $\begin{array}{l}\text { Error } \\
(\%)\end{array}$ & Q20 (\%) & Q30 (\%) & $\begin{array}{l}\text { GC content } \\
(\%)\end{array}$ \\
\hline $\mathrm{DC} 1 \_S 1^{a}$ & 57252114 & 55277910 & $8.29 \mathrm{G}$ & 0.01 & 97.53 & 94.05 & 46.16 \\
\hline $\mathrm{DC} 1 \_\mathrm{S} 2^{a}$ & 44863528 & 43312840 & $6.5 \mathrm{G}$ & 0.01 & 97.56 & 94.09 & 45.75 \\
\hline $\mathrm{DF} 1 \_\mathrm{S} \mathbf{1}^{b}$ & 58455694 & 56340260 & $8.45 \mathrm{G}$ & 0.01 & 97.37 & 93.7 & 46.2 \\
\hline DF1_S $2^{b}$ & 52912596 & 50959096 & $7.64 \mathrm{G}$ & 0.01 & 97.69 & 94.33 & 47.63 \\
\hline DC9_S $1^{c}$ & 47092876 & 45414294 & $6.81 \mathrm{G}$ & 0.01 & 97.25 & 93.47 & 46.48 \\
\hline DC9_S $2^{c}$ & 46537374 & 44964612 & $6.74 \mathrm{G}$ & 0.01 & 97.49 & 93.96 & 46.59 \\
\hline DF9_S $1^{d}$ & 53471134 & 51541182 & $7.73 \mathrm{G}$ & 0.01 & 97.54 & 94.09 & 47.3 \\
\hline DF9_S $2^{d}$ & 69564340 & 67080588 & $10.06 \mathrm{G}$ & 0.01 & 97.55 & 94.12 & 46.94 \\
\hline
\end{tabular}

${ }^{a}$ DC1_S1 \& DC1_S2: two biological replicates of the 1 week-old control group. ${ }^{b}$ DF1_S1 and DF1_S2: two biological replicates of the 1 week-old model group. ${ }^{c}$ DC9_S1 \& DC9_S2: two biological replicates of the 9 week-old control group. ${ }^{d}$ DF9_S1 \& DF9_S2: two biological replicates of the 9 week-old model group.

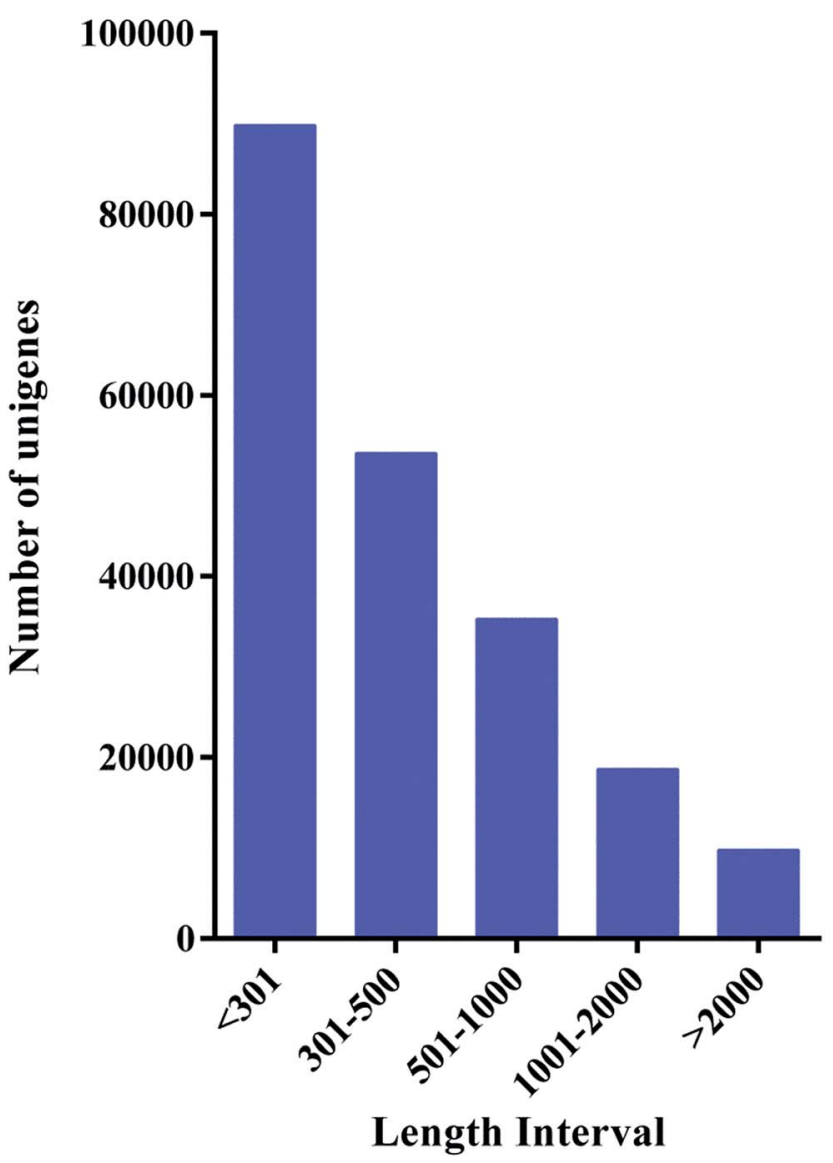

Fig. 3 Length distribution of the unigenes.

the changes in polysaccharide content between symbiotic and asymbiotic $D$. nobile were not significant at this time point; these pathways included the citrate cycle (TCA cycle), carbon metabolism, carbon fixation in photosynthetic organisms, the pentose phosphate pathway and glycolysis/gluconeogenesis. These results suggested that the effect of MF23 on the polysaccharide metabolism of $D$. nobile began at the early stage of symbiotic culture, even before the hyphae touched the roots.

\section{Unigenes involved in polysaccharide accumulation}

Focusing on the pathways involved in polysaccharide biosynthesis and consumption led to the identification of 6 DEGs from DF9_S vs. DC9_S, including key genes for photosynthesis (ferredoxin (petF), cytochrome c6 (petJ)), starch and sucrose metabolism (sucrose synthase (SUS), cellulose synthase (UDPforming) (bcsA), starch synthase $(g \lg A))$ and glycolysis (phosphoglycerate kinase (PGK)) (Table 2). Furthermore, since apart from glucose, mannose is the main monosaccharide component of $D$. nobile polysaccharides, ${ }^{46}$ and because respiration is the major consumer of polysaccharides, the unigenes encoding key enzymes of mannose biosynthesis and respiration were identified by searching for the names of the annotated genes. A total of 5 unigenes involved in fructose and mannose metabolism were found, including one encoding phosphomannomutase (PMM) and 4 annotated to mannose-1-phosphate guanylyltransferase (GMPP) (ESI Table 2†). Regarding the pathways associated with respiration, the genes involved in the citrate cycle and glycolysis, including the pyruvate dehydrogenase $\mathrm{E} 1$ component beta subunit $(P D H B)$, the pyruvate dehydrogenase E2 component (dihydrolipoamide acetyltransferase; $D L A T)$, citrate synthase (CS), the succinate dehydrogenase (ubiquinone) flavoprotein subunit (SDHA), and 6-phosphofructokinase 1 (PFK), were mined (Fig. 8 and ESI Table $2 \dagger$ ).

\section{Validation and expression analysis of genes involved in polysaccharide accumulation}

In this study, 13 key enzyme-encoding genes associated with polysaccharide accumulation, including both the biosynthetic and consumption pathways, were chosen for qRT-PCR analysis. Except for petJ and $P M M$, all chosen key enzyme-coding genes involved in polysaccharide biosynthesis exhibited $>2$-fold higher expression in D. nobile in response to MF23 than in the control at the $9^{\text {th }}$ week, when the content of polysaccharides was high (Fig. 9). In contrast, the expression levels of most genes associated with polysaccharide consumption were not significantly changed at 9 weeks (Fig. 10), similar to the result obtained using Illumina sequencing technology, which indicated that our experimental results were valid. 
E-value Distribution

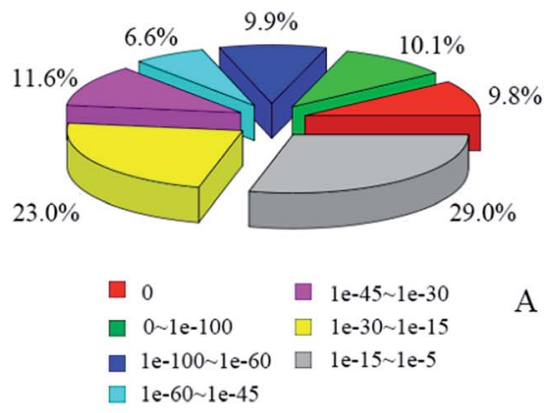

Similarity Distribution
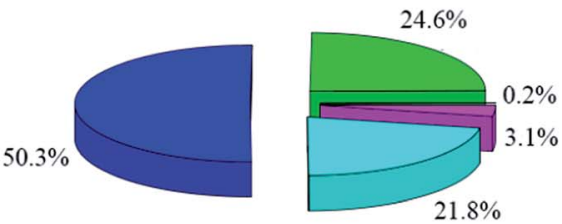

$\quad 18 \% \sim 40 \%$
$\square \quad 40 \% \sim 60 \%$
$\square \quad 60 \% \sim 80 \%$

$80 \% \sim 95 \%$

— $95 \% \sim 100 \%$
B

\section{Species classification}

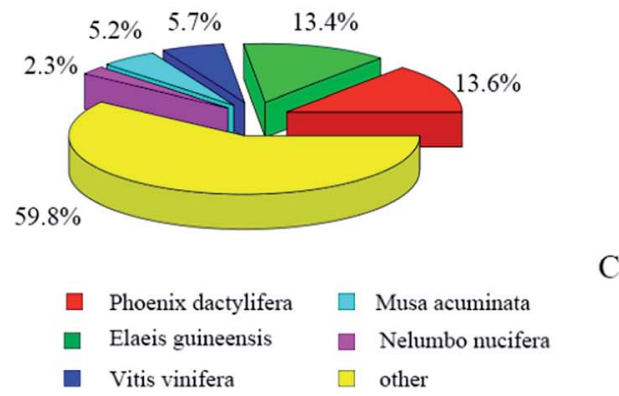

Fig. 4 Characteristics of similarity search of unigenes against the NR database: (A) $E$-value distribution of BLAST hits for each unigene with a cutoff $E$-value of $10^{-5}$; (B) similarity distribution of the top BLAST hit for each unigene; (C) species distribution of the top BLAST hit for each unigene in the NR database.

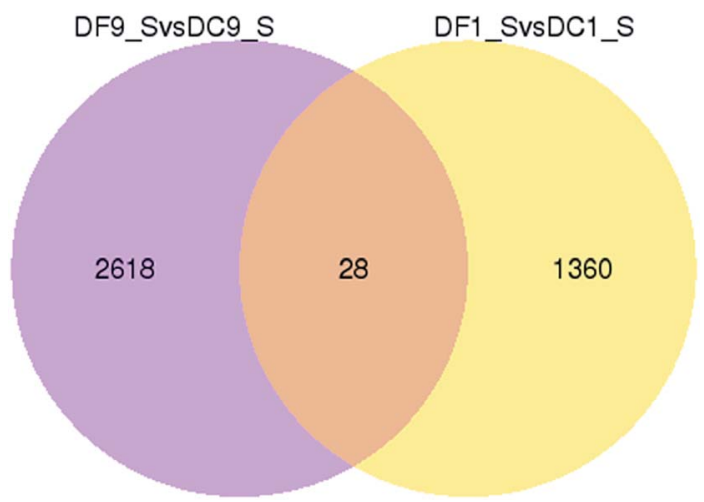

Fig. 5 Venn diagram of differentially expressed genes in symbiotic and asymbiotic samples. " $X$ vs. $Y$ " means $Y$ is the control.

In addition, the expression level of $p e t F$, which encodes a key enzyme of photosynthesis, was markedly repressed before 9 weeks, while genes involved in respiration, $P D H B, C S, S D H A$ and $P F K$, were activated to different degrees at different stages. This finding implied that $D$. nobile consumed more polysaccharides in the early period after induction by MF23.

\section{Discussion}

Polysaccharides are among the main active constituents of $D$. nobile. Because of the positive effects of these constituents on human health, research on $D$. nobile polysaccharides has been of great interest, especially studies on their content, ${ }^{\mathbf{4 7}}$ composition $^{\mathbf{4 8 , 4 9}}$ and pharmacological effects. ${ }^{50}$ However, with the exception of two reports by Guo's research group, ${ }^{23,24}$ there are few reports on technology that can elevate the polysaccharide content of $D$. nobile or on the molecular mechanisms by which its polysaccharide content increases. In this study, we assessed the possible mechanism of the MF23-mediated induction of polysaccharide content, which helps us to understand the relationship between a mycorrhizal fungus and its host and provides a new strategy to promote the active components of $D$. nobile.

Pelotons indicate the formation of orchid mycorrhizae. A previous isotope feeding experiment showed that in mycorrhizae, $\mathrm{C}$ flows into senescent pelotons and is then transferred to the host. ${ }^{51}$ In some respects, pelotons are considered pools of nutrition and centres of nutrition transformation that deliver nutrients from the host and environment to the energydissipating area of plant. ${ }^{52}$ Thus, MF23 might promote the biosynthesis of polysaccharides by forming pelotons to supply nutrition to its host, $D$. nobile.

A mycorrhizal fungus may influence its host even before colonization. Many studies have shown that the secretions of mycorrhizal fungi can rapidly and specifically induce the expression of specific genes in medicinal plants, activating a series of specific secondary metabolic pathways. ${ }^{53,54}$ For instance, when cultured on MS medium supplied with 
Enriched GO Terms

(DF1_S vs DC1_S)

BP

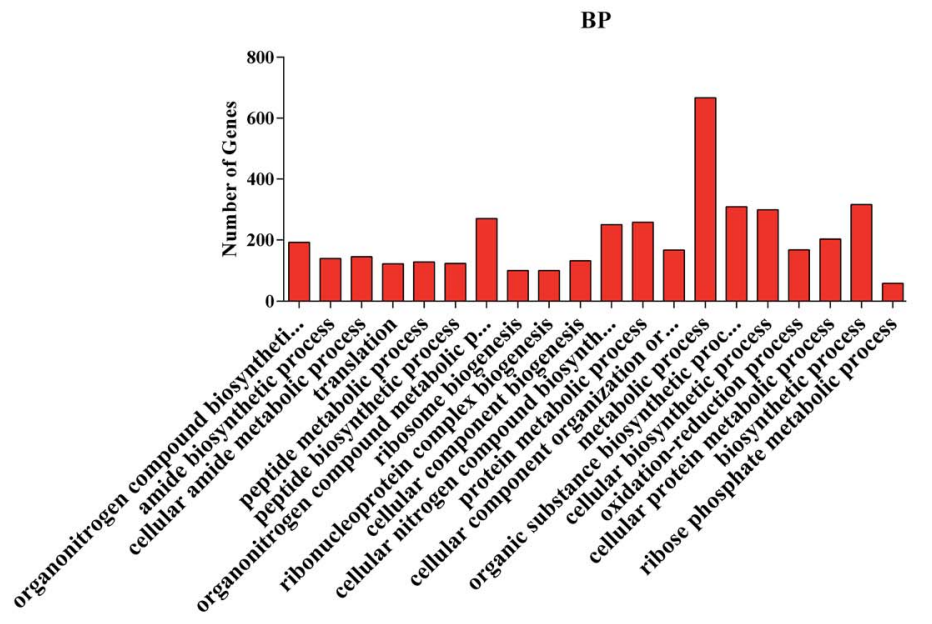

MF

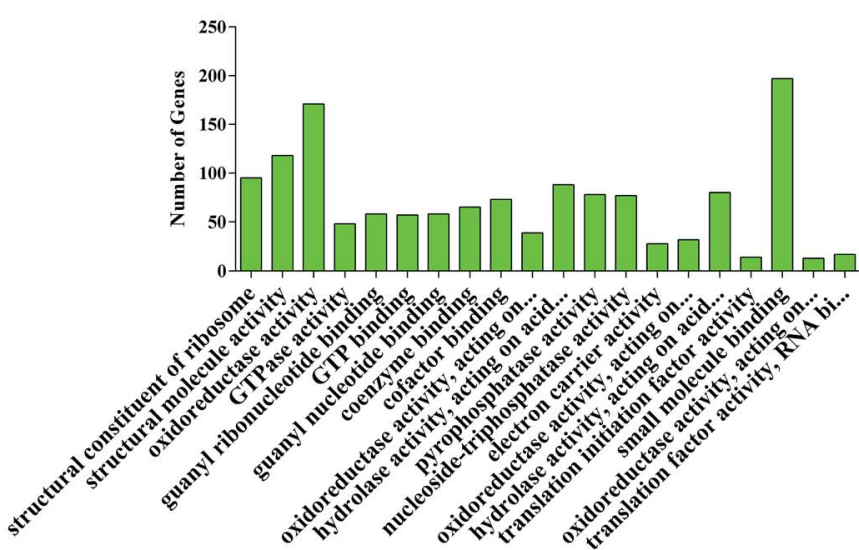

Enriched GO Terms

(DF9_S vs DC9_S)

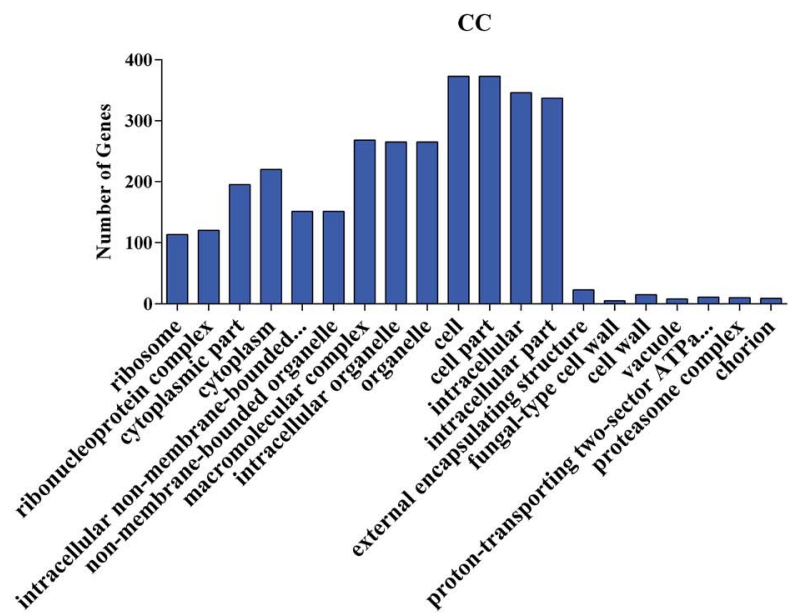

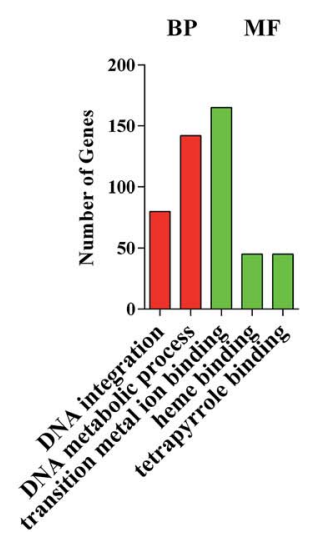

Fig. 6 GO functional classification of differentially expressed genes. BP, biological process, CC, cellular component, MF, molecular function.

fermentation broths of mycorrhizal fungus Th-9.7-1, the expression of expansin gene in Tetrastigma hemsleyanum was significantly up - regulated (>3-fold), though there was no fungal colonization..$^{55}$ The similar pattern was also found in the present study during the early period of MF23 infection. Although there was no significant change in the light 
DF1_S vs DC1_S

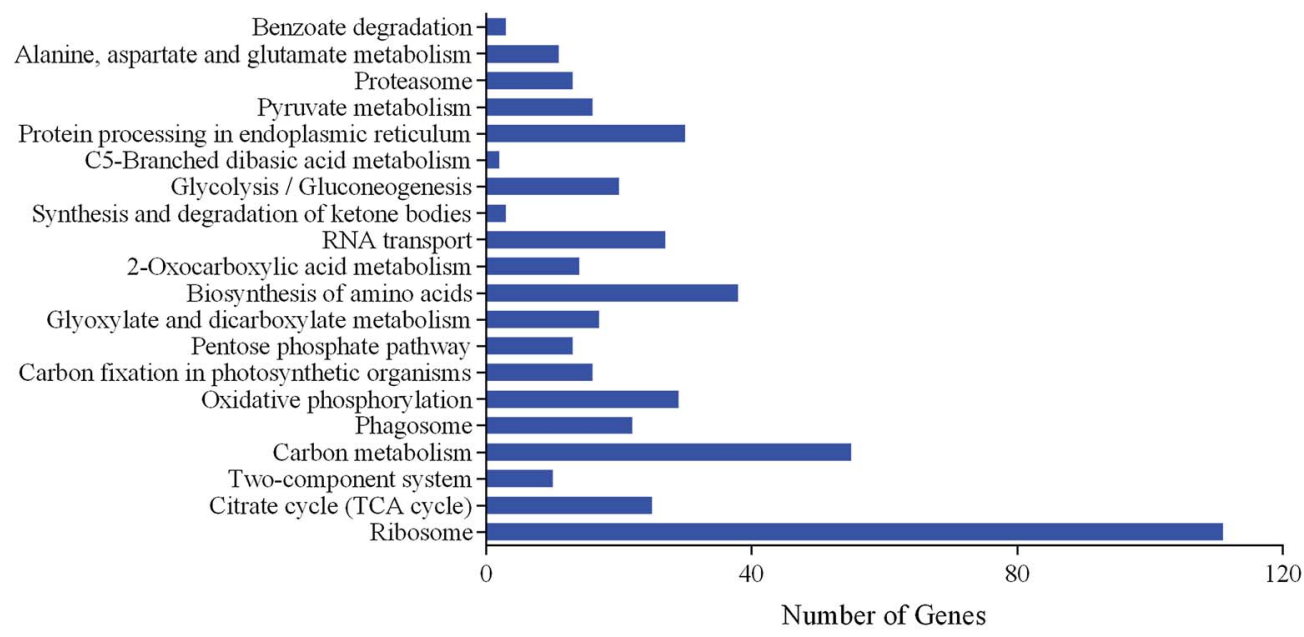

DF9_S vs DC9_S

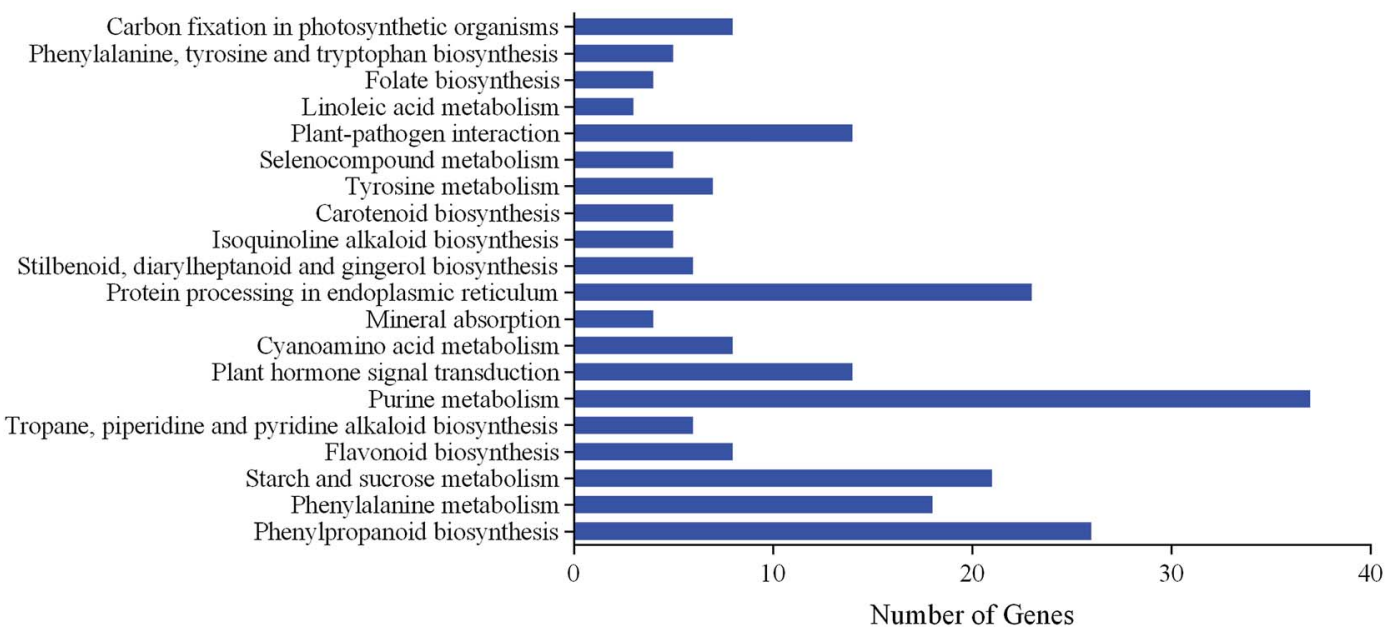

Fig. 7 Summary of the top 20 KEGG pathways of DEGs.

microscopy and the polysaccharide content in the $1^{\text {st }}$ week after inoculation, a total of 1388 DEGs were observed between DF1_S and DC1_S. In addition, 25\% of the top 20 KEGG pathways in the $1^{\text {st }}$ week were involved in polysaccharide metabolism. These results indicate that the effect of MF23 on the polysaccharide metabolism of $D$. nobile occurred much earlier than colonization. MF23 may release a 'fungal elicitor $^{56,57}$ into the culture medium to produce physiological changes in $D$. nobile and then promote polysaccharide metabolism.

Table 2 DEGs (DF9_S vs. DC9_S) involved in polysaccharide accumulation

\begin{tabular}{|c|c|c|c|c|c|}
\hline $\begin{array}{l}\text { Direction of } \\
\text { carbon flow }\end{array}$ & Pathway & Gene name & Unigene ID & $\log _{2}$ fold $^{2}$ & Padj $^{3}$ \\
\hline \multirow[t]{3}{*}{ Biosynthesis } & Photosynthesis & Ferredoxin (petF) & c72738_g2 & 1.4667 & 0.016632 \\
\hline & Starch and sucrose & Sucrose synthase (SUS) & c89528_g2 & 6.2099 & $8.74 \times 10^{-9}$ \\
\hline & metabolism & $\begin{array}{l}\text { Cellulose synthase } \\
\text { (UDP-forming) }(b c s A)\end{array}$ & c92372_g2 & 1.067 & 0.042397 \\
\hline
\end{tabular}




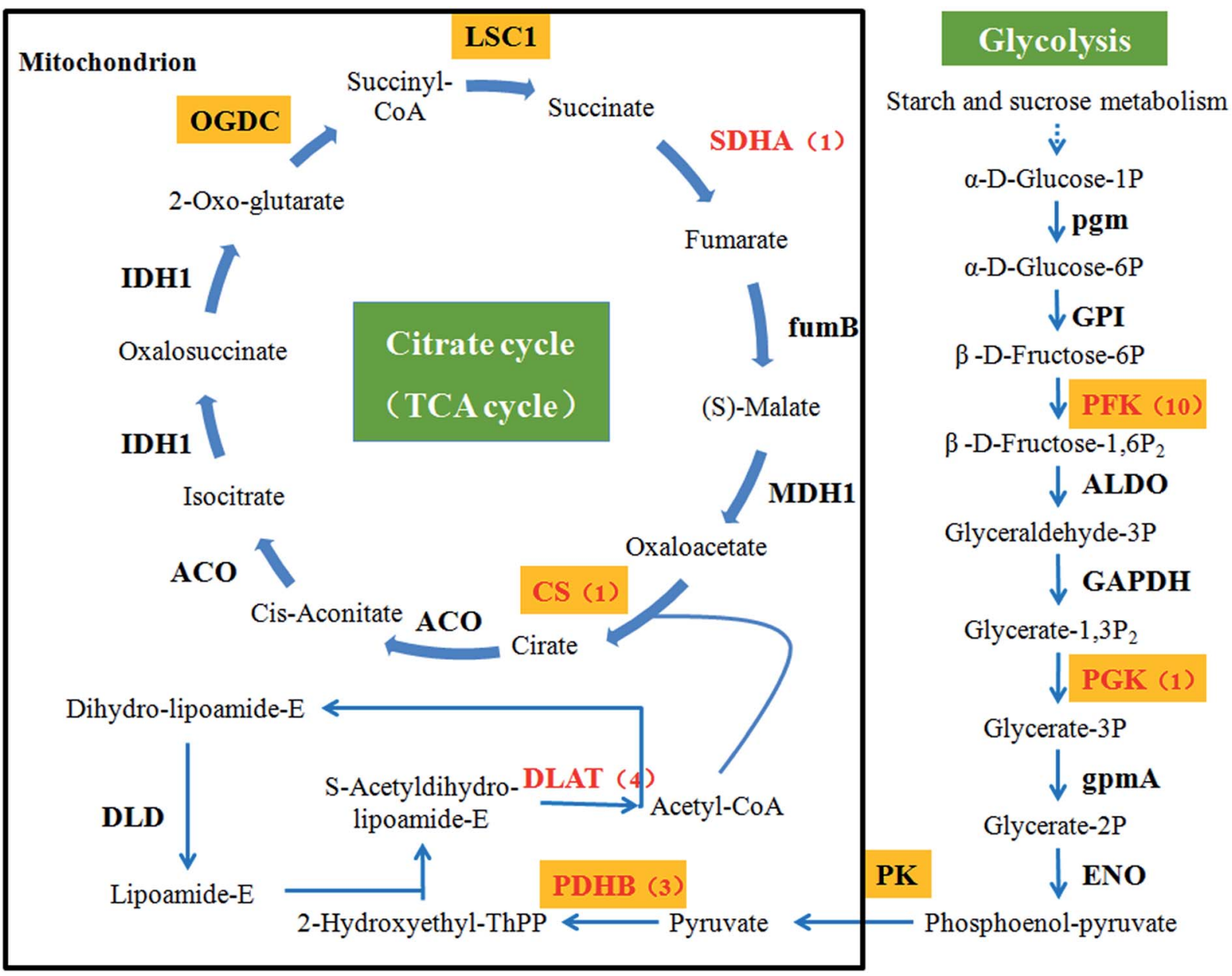

Fig. 8 Glycolysis and the citrate cycle (TCA cycle). Gene names with an orange background indicate that the gene product catalyses an irreversible reaction. The genes that we identified via RNA-Seq are highlighted in red. The numbers in brackets following the gene names indicate the number of unigenes annotated to each gene.

Photosynthesis is the main pathway by which plants obtain carbohydrates. Among the key enzymes involved in photosynthesis, petF, the terminal electron acceptor of photosystem I (PSI) seems more important. ${ }^{58}$ It delivers electrons to FDXNADPH-oxidoreductase (FNR) to generate NADPH, which is required for carbon assimilation in the Calvin cycle..$^{59}$ Furthermore, evidence has shown that Thalassiosira oceanica expressing exogenous petF has increased tolerance to severe environments by maintaining the electron transport interface of photosynthesis intact. ${ }^{60}$ Since polysaccharides are the main carbohydrates in plant, petF plays a vital role in the biosynthesis of polysaccharides. In this study, the expression of petF markedly increased by more than 6-fold in symbiotic D. nobile 9 weeks after inoculation, when the content of polysaccharides was elevated; prior to this time point, petF expression was at a very low level. These findings strongly support the importance of petF and imply that MF23 may stimulate polysaccharide biosynthesis by activating the expression of petF to assimilate more carbon.

Cellulose contributes to the structural support of the cell wall and is the most common polysaccharide in plants. ${ }^{61}$ The expression of $b c s A$, which encodes cellulose synthase, was clearly and significantly induced (over 4-fold) in the model group in the $9^{\text {th }}$ week. This induction might be one of the most important reasons for the accumulation of polysaccharides. Interestingly, although the expression levels of $b c s A$ in symbiotic D. nobile in the $1^{\text {st }}, 3^{\text {rd }}$, and $6^{\text {th }}$ weeks were lower than those in the $9^{\text {th }}$ week, they were higher than those of the control group all time points. The plant cell wall thickens in response to stress. ${ }^{62}$ During resistance responses, the secondary walls of the xylem in rice seedlings thicken within $48 \mathrm{~h}$, effectively reducing the area of the pit membrane that may be accessed by bacteria. ${ }^{63}$ Similarly, the induction of $b c s A$ in symbiotic $D$. nobile might also be associated with plant defence mechanisms.

SUS is one of the key enzymes of sucrose metabolism, primarily affecting the degradation of sucrose to provide precursors for polysaccharides. ${ }^{64}$ For Anabaena sp., the accumulation of polysaccharide in a mutant strain constitutively overexpressing SUS increase to 2-fold more than those of the mutant strain with an insertion inactivation of SUS and the wild strain, which demonstrated the importance of SUS in the biosynthesis of polysaccharides. ${ }^{65}$ In recent years, many studies 


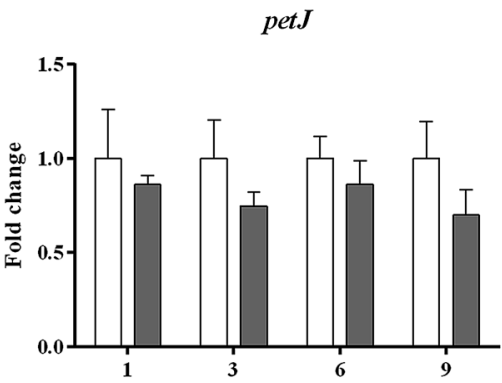

$S U S$

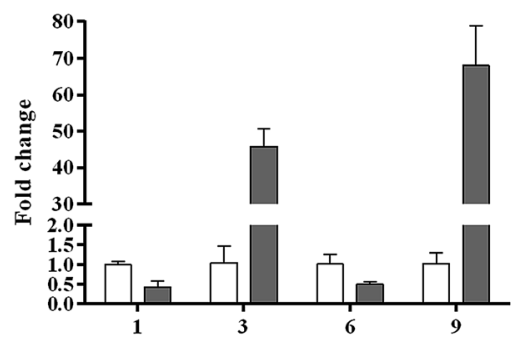

GMPP

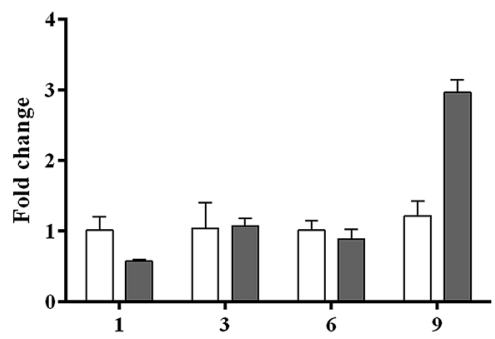

petF

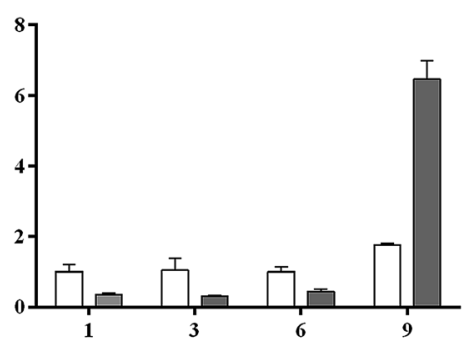

$\operatorname{glg} A$

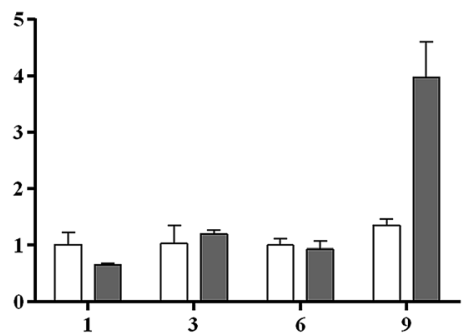

bcs A

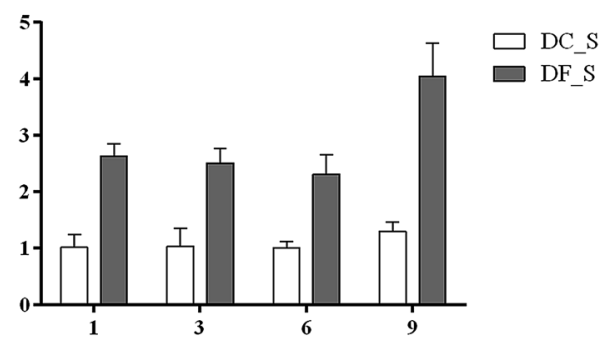

PMM

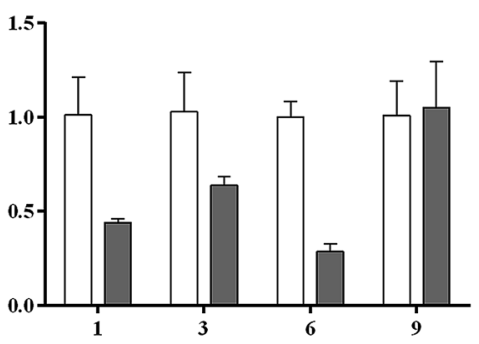

Fig. 9 qRT-PCR analysis of key enzyme-encoding genes involved in polysaccharide biosynthesis in $D$. nobile. The $y$-axis corresponds to the mean fold change in expression, and the $x$-axis displays the time in symbiotic culture (/weeks). The white bar represents the control group (DC_S), and the grey bar represents the model group (DF_S).

have indicated that for D. huoshanense and D. officinale, an increase in the polysaccharide content was always accompanied by the activation of SUS. ${ }^{66-68}$ Therefore, in D. nobile, SUS may play the same role in polysaccharide biosynthesis is in these related plant species. This conjecture was supported by the 68.06-fold higher expression of SUS over that in the controls in the $9^{\text {th }}$ week. This result means that MF23 might increase the polysaccharide content by activating the expression of SUS.

Starch is the final product of photosynthesis, and it stores energy from photosynthesis for many types of metabolism. Starch is complex. On the one hand, starch itself is a polysaccharide; on the other hand, starch can be degraded to glucose, the main constituent of different types of polysaccharides. Regardless of the role of starch, its accumulation has a positive impact on the accumulation of polysaccharides. Evidence has suggested a positive correlation between the contents of starch and polysaccharides in Dendrobium. ${ }^{69}$ Thus, the effect of the starch synthase gene $g l g A$ on polysaccharide biosynthesis is non-negligible. In this study, the expression of $g \lg A$ rose sharply in the $9^{\text {th }}$ week when the polysaccharide content was high, implying that $g l g A$ plays a vital role in the influence of MF23 on polysaccharide biosynthesis.
By using precolumn derivation HPLC, more than $60 \%$ monosaccharice compositions in polysaccharide of D. nobile were identified as mannose, which implied that mannosecontaining polysaccharides are main types of polysaccharides in D. nobile. ${ }^{46} \mathrm{GDP}-\mathrm{mannose}$ is a donor for the biosynthesis of mannose-containing polysaccharides. ${ }^{70}$ PMM catalyses the conversion of mannose-6-phosphate to mannose-1-phosphate, which is the precursor for the biosynthesis of GDP-mannose. ${ }^{71}$ GMPP catalyses the formation of GDP-mannose directly. ${ }^{72}$ Thus, changes in $P M M$ and GMPP might make a substantial difference in the proportion of mannose-containing polysaccharides in D. nobile and lead to an increase in polysaccharide accumulation. In this study, the expression level of $P M M$ was significantly repressed at the first three stages, but returned to the control level in the $9^{\text {th }}$ week after inoculation. This result suggested that $P M M$ might play an indirect but essential role in the regulation of polysaccharide biosynthesis in symbiotic $D$. nobile. Unlike $P M M, G M P P$ was expressed at a high level in response to MF23 when the polysaccharide content was high. This corresponding relationship demonstrated that MF23 might increase the polysaccharide content by stimulating the expression of GMPP. 
$D L A T$

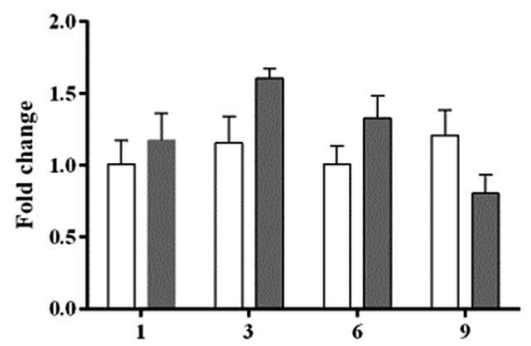

$S D H A$

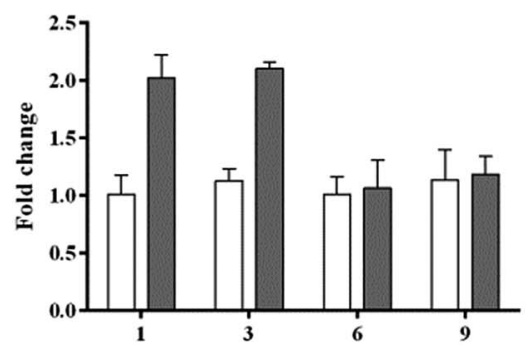

PDHB

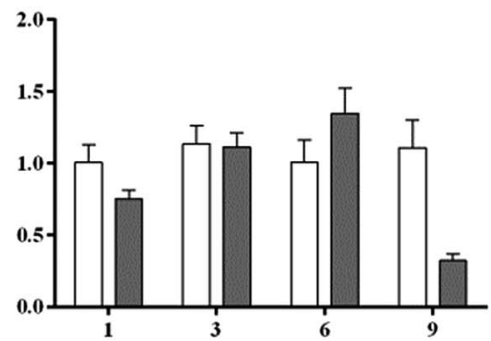

PFK

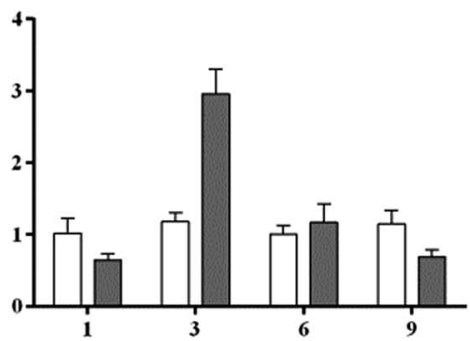

$C S$
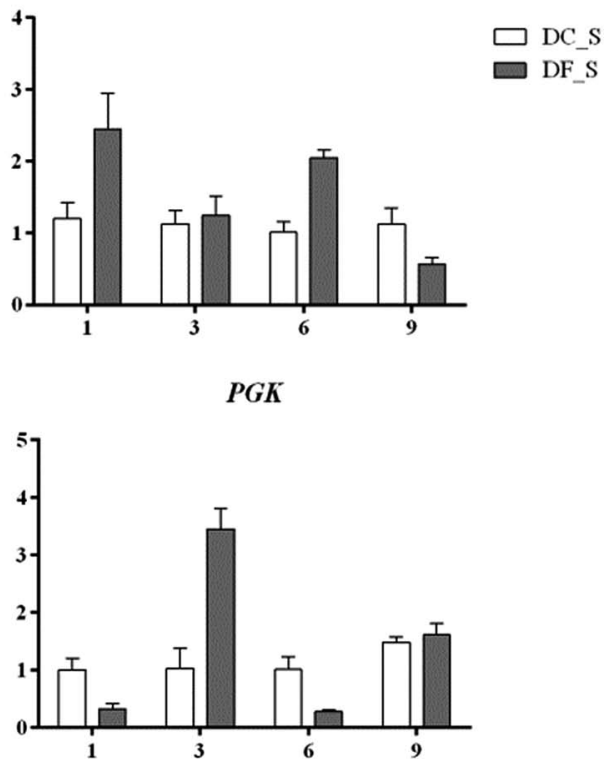

Fig. 10 qRT-PCR analysis of key enzyme-encoding genes involved in polysaccharide consumption in $D$. nobile. The $y$-axis corresponds to the mean fold change in expression, and the $x$-axis displays the time in symbiotic culture (/weeks). The white bar represents the control group (DC_S), and the grey bar represents the model group (DF_S).

As shown above, nearly all the genes that we chose that were involved in the polysaccharide biosynthesis pathway were upregulated in the $9^{\text {th }}$ week. However, polysaccharide accumulation also depends on the carbohydrate consumption rates, which are affected by glycolysis and the citrate cycle. Early studies showed that the exogenous expression of $P F K$, encoding a key enzyme of the glycolysis pathway, reduced the sugar content of potato tubers. ${ }^{73}$ For higher organisms, there is a positive correlation between the respiration rate and the enzyme activity in the citrate cycle. ${ }^{74-76}$ In this study, we assessed the relationship between 6 key genes in these two pathways, including DLAT, PDHB, CS and SDHA, PFK and PGK, as well as the polysaccharide content (Fig. 8). No genes were markedly induced in the $9^{\text {th }}$ week. Instead, $P D H B$ was significantly downregulated to levels 3.46-fold lower than those of controls. These results suggested that the consumption of polysaccharides was at a normal or low level in the $9^{\text {th }}$ week. Thus, these results that the activation of genes involved in polysaccharide biosynthesis in the $9^{\text {th }}$ week might be the main reason for polysaccharide levels to increase in D. nobile infected with MF23.

Before the $9^{\text {th }}$ week, $C S, S D H A, P F K$ and $P G K$ in symbiotic $D$. nobile were affected to different degrees at different stages. For instance, the expression levels of $S D H A, P F K$ and $P G K$ were upregulated more than 2 -fold in the $3^{\text {rd }}$ week, implying that MF23 might influence the rate of respiration at the early stage of inoculation; this result was in accordance with the results from Cymbidium sinense and C. ensifolium infected with a mycorrhizal fungus. ${ }^{77} \mathrm{CS}$, PFK and PGK catalyse the pivotal irreversible reaction that leads to the consumption of carbohydrates. ${ }^{78}$ SDHA participates in both the citrate cycle and the electron transport chain. ${ }^{79}$ Their stimulation would undoubtedly result in a reduction in polysaccharides. However, there was no significant change in the polysaccharide content before the $9^{\text {th }}$ week. A further analysis of recent experimental data showed that the high expression levels of two key genes, SUS and bcsA, which were associated with starch and sucrose metabolism before the $9^{\text {th }}$ week, might account for this lack of change. These findings strongly suggested that MF23 might influence the polysaccharide content by regulating both the biosynthesis and consumption pathways of its host and that the combination of the effects of these two pathways determines the accumulation of polysaccharides. However, the polysaccharide metabolic network is complex, and further research regarding the mechanisms that regulate it should be performed.

\section{Conclusions}

To determine the mechanism by which polysaccharide accumulation is regulated in $D$. nobile infected with the mycorrhizal fungus MF23, the first large-scale transcriptome dataset from $D$. nobile (control and model groups) was generated using RNASeq. Using the results of RNA-Seq and qRT-PCR, we showed that the regulatory network by which MF23 acts on D. nobile is complex. MF23 might regulate both the biosynthesis and consumption of polysaccharides to change polysaccharide accumulation. The combined effects of the activated biosynthetic pathway and the depressed consumption pathway might result in the accumulation of polysaccharides. Among the analysed genes, petF, SUS, bcsA, PMM, GMPP, PDHB, CS, SDHA, $P G K$ and $P F K$ might play vital roles in this regulatory network. This study provides a good example of an endophyte promoting the formation of bioactive compounds in its host and paves the way for further investigations of the associated regulatory mechanisms using molecular methods. 


\section{Acknowledgements}

The research was financially supported by the National Natural Science Foundation of China (No. 31170314, No. 81473331).

\section{Notes and references}

1 X. S. Bao, Q. S. Shun and C. L. Zhuan, The medicinal plants of dendrobium(shi-hu)in china a coloured atlas, Fudan University Press, Shanghai, 2001.

2 S. Chen, Y. Li, Y. Wu, Z. Zhou and L. Sun, China J. Chin. Mater. Med., 1995, 20, 181-182.

3 M. Y. Yoon, J. H. Hwang, J. H. Park, M. R. Lee, H. J. Kim, E. Park and H. R. Park, J. Med. Food, 2011, 14, 120.

4 Q. Huang, X. Liao, W. U. Qin, L. I. Fei, L. Y. Wang and J. S. Shi, Chin. J. New Drugs Clin. Rem., 2013, 32, 490-493.

5 M. M. Li, B. X. Zhang, S. B. He, R. Zheng, Y. L. Zhang and Y. Wang, China J. Chin. Mater. Med., 2015, 40, 3709-3712.

6 Q. Ye, G. Qin and W. Zhao, Phytochemistry, 2002, 61, 885-890.

7 Y. P. Li, J. H. Jiang, Y. Liu and Y. G. Chen, Lishizhen Med. Mater. Med. Res., 2010, 21, 39-40.

8 X. M. Zhang, Z. R. Sun, L. Chen, X. X. Wei and W. L. Liu, Chin. J. Mod. Appl. Pharm., 2014, 31, 895-899.

9 F. J. An and Y. X. He, J. Anhui Agric. Sci., 2014, 42, 3857-3862. 10 L. N. Wang, Q. H. Gong, F. Li, Q. Wu and J. S. Shi, Acta Neuropharmacologica, 2016, 6, 89.

11 C. J. Li, Yunnan Journal of Traditional Chinese Medicine and Meteria Medica, 2012, 33, 61-62.

12 Z. H. Wang, J. Li, J. H. Zang, Y. C. Xu and Q. S. Ye, Chinese Journal of Tropical Crops, 2016, 37, 2333-2337.

13 X. J. Ge, L. M. Zheng, Y. L. Wang and Y. P. Tang, Chongqing Med., 2015, 44, 1305-1310.

$14 \mathrm{~S} . \mathrm{Z}$. Zheng, Effects of the proliferation and apoptosis of $\mathrm{K562}$ cell affects by Dendrobium nobile polysaccharides, Zunyi Medical University, 2009.

15 A. Luo and Y. Fan, Afr. J. Pharm. Pharmacol., 2011, 5, 625631.

16 J. H. Wang, Carbohydr. Polym., 2010, 79, 114-118.

17 Y. Y. Xu, L. Y. Wang, B. Huang, X. L. Xie, Q. Wu and J. S. Shi, West China J. Pharm. Sci., 2014, 29, 288-291.

18 M. A. Selosse and M. Roy, Trends Plant Sci., 2009, 14, 64-70. 19 Q. X. Wang, N. Yan, D. G. Ji, S. Y. Li, J. M. Hu and H. Hu, Plant Diversity Resour., 2014, 36, 321-330.

20 L. Q. Chen, X. M. Wang and Z. D. Pei, Forest Res., 2005, 18, 163-168.

21 L. C. Zhang and S. X. Guo, J. Chin. Pharm. Sci., 2009, 44, 1540-1543.

22 J. Chen, K. X. Hu, X. Q. Hou and S. X. Guo, World J. Microbiol. Biotechnol., 2011, 27, 1009-1016.

23 H. L. Yan, X. M. Chen, F. H. Liao, X. F. Deng, C. L. Wang, L. X. Tian, S. X. Guo and J. Y. Yang, Chin. Pharm. J., 2016, 51, 1450-1454.

24 X. M. Chen and S. X. Guo, China J. Chin. Mater. Med., 2005, 30, 253-257.

25 Y. L. Wang, W. H. Chen, Y. X. Xie, J. Xu and J. Wang, Soil Fert. Sci. China, 2000, 32-34.
26 Z. Y. Chen, Correlation between polysaccharides accumulation and sugar metabolism in Dendrobium officinale, Zhejiang A \& F University, 2015.

27 F. C. Zhao, L. Q. Jing, D. L. Lu, G. Y. Wang and W. P. Lu, J. Nucl. Agric. Sci., 2014, 28, 2230-2237.

28 X. C. Liu and A. Vonshak, J. Guangxi Norm. Univ., 2003, 21, 91-95.

29 L. Zhang, J. Chen, Y. Lv, C. Gao and S. Guo, Mycol. Progr., 2012, 11, 395-401.

30 X. M. Chen, H. L. Yan, C. L. Wang, L. X. Tian, A. R. Wang and S. X. Guo, Sci. China: Life Sci., 2016, 59, 974-976.

31 N. P. C., Pharmacopoeia of the People's Republic of China, China Medical Science Press, 2015, vol. 1, pp. 282-283.

32 M. Dubois, K. A. Gilles, J. K. Hamilton, P. A. Rebers and F. Smith, Anal. Chem., 1956, 28, 350-356.

33 N. Feder and T. P. O'Brien, Am. J. Bot., 1968, 55, 123-142.

34 M. M. Liu, Y. M. Xing, D. W. Zhang and S. X. Guo, Sci. Rep., 2015, 5, 16075.

35 M. Pei, J. Niu, C. Li, F. Cao and S. Quan, BMC Genomics, 2016, 17, 132.

36 M. G. Grabherr, B. J. Haas, M. Yassour, J. Z. Levin, D. A. Thompson, I. Amit, X. Adiconis, L. Fan, R. Raychowdhury and Q. Zeng, Nat. Biotechnol., 2011, 29, 644-652.

37 L. Wang, Z. Feng, X. Wang, X. Wang and X. Zhang, Bioinformatics, 2010, 26, 136.

38 J. D. Storey and R. Tibshirani, Proc. Natl. Acad. Sci. U. S. A., 2003, 100, 9440-9445.

39 M. D. Young, M. J. Wakefield, G. K. Smyth and A. Oshlack, Genome Biol., 2010, 11, 1-12.

40 X. Mao, T. Cai, J. G. Olyarchuk and L. Wei, Bioinformatics, 2005, 21, 3787.

41 W. Chen, X. Cheng, Z. Zhou, J. Liu and H. Wang, Mol. Biol. Rep., 2013, 40, 1145-1154.

42 K. J. Livak and T. D. Schmittgen, Methods, 2001, 25, 402-408.

43 G. Hadley, Mycorrhizas and plant growth and development, Springer, Netherlands, 1988.

44 M. Kanehisa, M. Araki, S. Goto, M. Hattori, M. Hirakawa, M. Itoh, T. Katayama, S. Kawashima, S. Okuda and T. Tokimatsu, Nucleic Acids Res., 2008, 36, D480-D484.

45 M. Kanehisa, Y. Sato, M. Kawashima, M. Furumichi and T. Mao, Nucleic Acids Res., 2016, 44, D457.

46 X. U. Li, L. Guo, F. L. Luo, T. M. Zhang, X. W. Liu and Y. Han, Lishizhen Med. Mater. Med. Res., 2014, 25, 1725-1727.

47 Y. Y. Wang, H. Xu, S. S. Shi, L. S. Wang and Z. T. Wang, J. Chin. Med. Mater., 2009, 32, 493-495.

48 A. X. Luo, Carbohydr. Polym., 2010, 79, 1014-1019.

49 J. H. Wang, Carbohydr. Polym., 2010, 81, 1-7.

50 Y. L. Zhang, X. R. He, Y. Y. Liu, L. X. Qiong and Y. P. Tang, Lishizhen Med. Mater. Med. Res., 2013, 24, 7-9.

51 Y. Kuga, N. Sakamoto and H. Yurimoto, New Phytol., 2014, 202, 594-605.

52 R. L. Wang, H. Hu and S. Y. Li, Acta Bot. Yunnanica, 2004, 26, 445-450.

53 X. Zhai, M. Jia, L. Chen, C. J. Zheng, K. Rahman, T. Han and L. P. Qin, Crit. Rev. Microbiol., 2016, 43, 238-261. 
54 Y. Tan, R. Jia, J. H. Tao, N. Y. Yang, J. A. Duan, D. W. Qian and S. Jiang, Chin. Tradit. Herb. Drugs, 2013, 44, 20042008.

55 Y. L. song, Molecular cloning of expansion gene, isolation and identification of emdophytic fungi from root tuber of Tetrastigma hemsleyanum, Hangzhou Normal University, 2016.

56 X. M. Chen, S. X. Guo and Z. X. Meng, Chin. Pharm. J., 2006, 41, 423-426.

57 J. H. Chen, C. Wang, L. Shi and J. M. Yin, Hunan Agricultural Sciences, 2014, 28-31.

58 B. Munge, S. K. Das, R. Ilagan, Z. Pendon, J. Yang, H. A. Frank and J. F. Rusling, J. Am. Chem. Soc., 2003, 125, 12457.

59 K. Fukuyama, Photosynth. Res., 2004, 81, 289-301.

60 M. Lommer, A. S. Roy, M. Schilhabel, S. Schreiber, P. Rosenstiel and J. Laroche, BMC Genomics, 2010, 11, 718.

61 D. L. Song, J. H. Shen and L. G. Li, Plant Physiol. Commun., 2008, 44, 791-796.

62 G. H. Le, F. Philippe, J. M. Domon, F. Gillet, J. Pelloux and C. Rayon, Plants, 2015, 4, 112-166.

63 E. Hilaire, S. A. Young, L. H. Willard, J. D. Mcgee, T. Sweat, J. M. Chittoor, J. A. Guikema and J. E. Leach, Mol. PlantMicrobe Interact., 2001, 14, 1411-1419.

64 S. Chalivendra, S. C. Huber, M. M. Sachs and D. Rhoads, Plant Signaling Behav., 2007, 2, 28-29.

65 L. Curatti, L. E. Giarrocco, A. C. Cumino and G. L. Salerno, Planta, 2008, 228, 617-625.

66 M. Wei, S. T. Jiang and J. P. Luo, Biotechnol. Lett., 2007, 29, 495-499.
67 J. Yang, H. L. Meng, S. C. Yang, W. Zhang, Y. H. Zha and G. S. Wen, J. West China For. Sci., 2012, 41, 62-67.

68 M. Wei, S. T. Jiang and J. P. Luo, Chin. J. Biotechnol., 2007, 23, 327-331.

69 H. Zhu, J. Teng, Y. Cai, J. Liang, Y. Zhu and T. Wei, China J. Chin. Mater. Med., 2011, 36, 3262-3264.

70 C. M. He, J. X. Zhang, C. Wang, K. L. Wu, S. J. Zeng and J. Duan, The Congress of Botanical Society of China and the 80th Anniversary, 2013.

71 C. He, S. Zeng, J. A. T. D. Silva, Z. Yu, J. Tan and J. Duan, Protoplasma, 2016, 1-12.

72 T. K. Hyun, J. D. Lim, J. K. Kim, E. S. Seong, J. G. Lee, B. S. Yoon, M. J. Kim, D. H. Cho and C. Y. Yu, Br. J. Orthod., 2005, 13, 155-158.

73 O. Navrátil, P. Bucher and J. Vacek, Czech J. Genet. Plant Breed., 2012, 48, 42-45.

74 L. J. Liu, J. Cang, H. W. Li, j. Yu, X. Wang, J. F. Wang, R. Huang and C. Xu, J. Triticeae Crops, 2013, 33, 65-72.

75 A. Nunes-Nesi, L. J. Sweetlove and A. R. Fernie, Physiol. Plant., 2007, 129, 45-56.

76 X. G. Zhu, Photosynthetic carbon assimilation and respiration, Higher Education Press, Beijing, 2012.

77 C. Pan, R. Chen and L. Li, J. Trop. Subtrop. Bot., 2002, 10, 4650.

78 R. C. Pan, X. J. Wang and N. H. Li, Plant Physiology, Higher Education Press, Beijing, 2004.

79 S. B. Huang, N. L. Taylor, E. Ströher, R. Fenske and A. H. Millar, Plant J., 2013, 73, 429-441. 